\title{
Rotatable anisotropy on ferromagnetic/ antiferromagnetic bilayer investigated by Brillouin light scattering
}

Cite as: J. Appl. Phys. 123, 043901 (2018); https://doi.org/10.1063/1.5009461

Submitted: 16 October 2017 . Accepted: 22 December 2017 . Published Online: 23 January 2018

R. L. Rodríguez-Suárez, A. B. Oliveira (D), F. Estrada, D. S. Maior, M. Arana (D), O. Alves Santos, A. Azevedo (iD, and S. M. Rezende
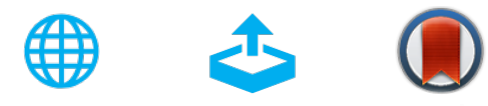

View Online

Export Citation

\section{ARTICLES YOU MAY BE INTERESTED IN}

Spin Seebeck effect in the antiferromagnet nickel oxide at room temperature Applied Physics Letters 111, 172405 (2017); https://doi.org/10.1063/1.5001694

Observation of magnons in $\mathrm{Mn}_{2} \mathrm{Au}$ films by inelastic Brillouin and Raman light scattering Applied Physics Letters 111, 192409 (2017); https://doi.org/10.1063/1.5001705

Conversion of spin current into charge current at room temperature: Inverse spin-Hall effect Applied Physics Letters 88, 182509 (2006); https://doi.org/10.1063/1.2199473

Lock-in Amplifiers Find out more today

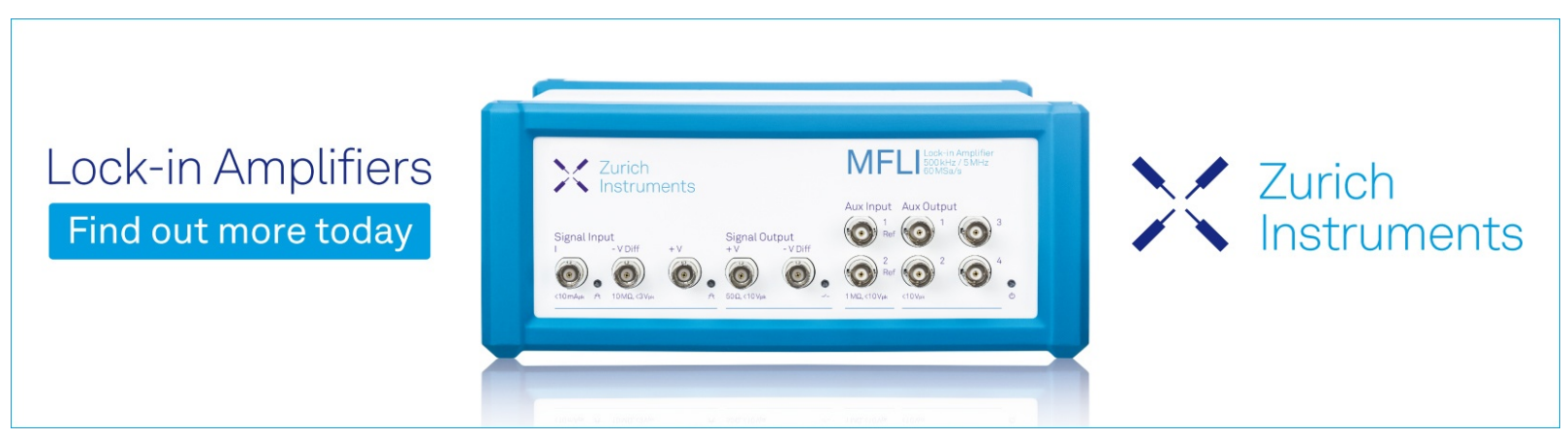

Instruments

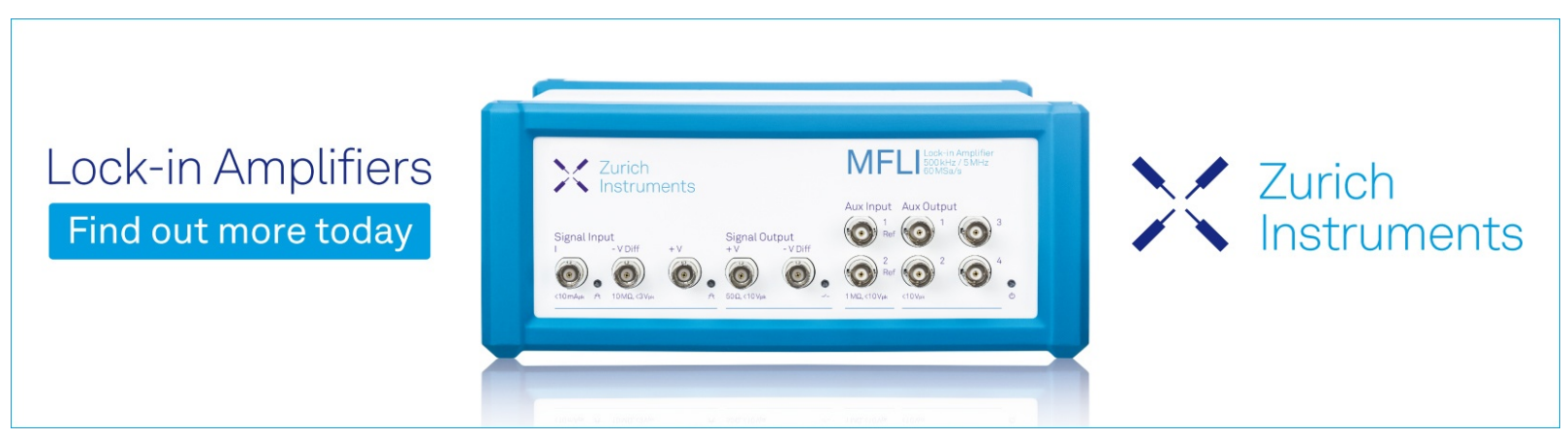




\title{
Rotatable anisotropy on ferromagnetic/antiferromagnetic bilayer investigated by Brillouin light scattering
}

\author{
R. L. Rodríguez-Suárez, ${ }^{1}$ A. B. Oliveira, ${ }^{2,3}$ F. Estrada, ${ }^{4,5}$ D. S. Maior, ${ }^{5}$ M. Arana,${ }^{5}$ \\ O. Alves Santos, ${ }^{5}$ A. Azevedo, ${ }^{5}$ and S. M. Rezende ${ }^{5}$ \\ ${ }^{1}$ Facultad de Física, Pontificia Universidad Católica de Chile, Av. Vicuña Mackenna 4860, Casilla 306, \\ Santiago, Chile \\ ${ }^{2}$ Departamento de Física Teórica e Experimental, Universidade Federal do Rio Grande do Norte, \\ 59072-970 Natal, RN, Brazil \\ ${ }^{3}$ Escola de Ciências \& Tecnologia, Universidade Federal do Rio Grande do Norte, 59072-970 Natal, RN, \\ Brazil \\ ${ }^{4}$ Facultad de Biología, Universidad Michoacana de San Nicolás de Hidalgo, Av. Francisco J. Mújica S/N, \\ Ciudad Universitaria, C. P. 58030, Morelia, Michoacán, Mexico \\ ${ }^{5}$ Departamento de Física, Universidade Federal de Pernambuco, 50670-901 Recife, Pernambuco, Brazil
}

(Received 16 October 2017; accepted 22 December 2017; published online 23 January 2018)

\begin{abstract}
The nature of the rotatable anisotropy in ferromagnetic/antiferromagnetic (FM/AF) NiFe/IrMn bilayers has been investigated using magneto-optical Kerr effect magnetometry, ferromagnetic resonance, and Brillouin Light Scattering (BLS) techniques. The behavior of the magnon frequencies with an external magnetic field applied in the bilayer plane has been measured by BLS. The angular dependence of magnon frequency shows a shift when compared to numerical calculation for different magnetic field intensities. This frequency shift can be associated with the rotatable anisotropy and it depends on the magnetic field intensity. As a result, the rotatable anisotropy field exhibits a non-monotonic dependence on the applied field. The results are interpreted considering the coexistence of parallel and antiparallel coupling at the FM/AF interface. Published by AIP Publishing.

https://doi.org/10.1063/1.5009461
\end{abstract}

\section{INTRODUCTION}

Although the exchange bias (EB) phenomenon, discovered 60 years ago, ${ }^{1}$ has been widely used in magnetoelectronic devices, ${ }^{2,3}$ the nature of the magnetic interactions at the ferromagnet/antiferromagnet (FM/AF) interface and its physical manifestations are still a prevalent problem in the area of thin film magnetism. Even more so when antiferromagnetic (AF) materials have received renewed attention in the emergent field of AF spintronics. ${ }^{4-6}$ The most known manifestation of the EB is the hysteresis loop shift $\left(H_{E}\right)$ along the field axis. Usually, the exchange bias direction is opposite (negative EB) to the direction of the magnetic field applied during sample growth. The reverse situation (positive EB), i.e., the shift of the hysteresis loop in the field direction is nowadays a known manifestation after it was observed 20 years ago in $\mathrm{Fe} / \mathrm{FeF}_{2}{ }^{7}$ and is usually associated with antiferromagnetic interfacial coupling at the FM/AF interface. ${ }^{8,9}$

Another important manifestation related to EB is the isotropic shift of the resonance field measured by ferromagnetic resonance (FMR). ${ }^{10-13}$ In order to explain it, two phenomenological models have been proposed by Stiles and McMichael $^{10,11,14,15}$ and Geshev et al. ${ }^{13}$ In both approaches, the main assumption is that there are two kinds of grains in the AF layer, one stable ("nonrotatable") and another unstable ("rotatable"), coupled with the adjacent FM magnetization. In the Stiles and McMichael model, the observed FMR field shift is explained introducing a unidirectional rotatable anisotropy (RA) term of the form $-\boldsymbol{M}_{F M} \cdot \boldsymbol{H}_{R A}$ in the AF part of the anisotropy energy, where $\boldsymbol{H}_{R A}$ is an effective magnetic field parallel to the equilibrium direction of the FM magnetization $\boldsymbol{M}_{F M}$. In the Geshev model, the rotatable anisotropy is introduced as a uniaxial anisotropy of the FM layer of the form, $-K_{r a}\left(\boldsymbol{M}_{F M} \cdot \hat{\boldsymbol{h}} / M_{F M}\right)^{2}$ with a symmetry axis along the applied field direction $\hat{\boldsymbol{h}}$. Such a kind of anisotropy should be used to model irreversible magnetization processes in which the directions of the FM magnetization and the applied magnetic field could differ considerably. Despite the different origins, both models give the same isotropic field shift result (same $H_{R A}$ ) under FMR measurements conditions, because at typical resonance fields the sample is saturated, and therefore, the FM magnetization and the applied field point in the same direction.

Typically, the observed isotropic shift of the FMR field $\left(H_{\text {res }}\right)$ is a downshift ${ }^{12,14,15}$ corresponding to a positive rotatable anisotropy (RA) field. Negative RA, i.e., the upward shift in the FMR field has been recently observed in $\mathrm{Co} / \mathrm{Cr}$ / IrMn, ${ }^{16} \mathrm{Co} / \mathrm{FeMn},{ }^{17}$ and $\mathrm{NiFe} / \mathrm{IrMn} .{ }^{18,19}$ Usually, rotatable anisotropy has been investigated by FMR in which measurements are limited to relatively high external fields ( $\sim 1000$ Oe). On the other hand, the Brillouin Light Scattering (BLS) technique has the advantage of working at constant magnetic fields, allowing the investigation of the field dependence of both anisotropy fields $H_{R A}$ and $H_{E}$.

In this paper, we report a novel behavior of the rotatable anisotropy in EB bilayers of $\mathrm{Ni}_{81} \mathrm{Fe}_{19}(7.5 \mathrm{~nm}) / \mathrm{Ir}_{20} \mathrm{Mn}_{80}\left(t_{A F}\right)$. We show that $H_{R A}$ has a non-monotonic dependence with the applied field. The BLS technique was used to measure the azimuthal angle dependence of the magnon frequency, while the applied field was fixed at 50 Oe, 200 Oe, and 1000 Oe. Through the angular dependence of $H_{r e s}$ and BLS frequency, 
we extract the anisotropy fields. The data were complemented by magnetization hysteresis measurements carried out by means of the Magneto-Optical Kerr Effect (MOKE) technique. Experimental results are discussed considering the coexistence of parallel and antiparallel coupling at the FM/AF interface. The results also indicate that the $\mathrm{AF}$ grain distribution between stable and unstable ones strongly depends on the applied field strength.

\section{EXPERIMENTS}

The FM/AF bilayers of $\mathrm{Ni}_{81} \mathrm{Fe}_{19}(7.5 \mathrm{~nm}) / \mathrm{Ir}_{20} \mathrm{Mn}_{80}\left(t_{A F}\right)$ used in this work were prepared by a dc magnetron sputtering technique. Prior to deposition of the IrMn layer, a $3 \mathrm{~nm}$ thick layer of $\mathrm{Ni}_{81} \mathrm{Fe}_{19}(\mathrm{Py})$ was grown on the $\mathrm{Si}(001)$ substrates. During the deposition of the seed layer of Py, we applied an in-plane magnetic field of 200 Oe strong enough to induce a uniaxial magnetic anisotropy. Then, the AF/FM bilayer was grown on top of the seed layer. By means of this procedure, a magnetic-textured layer of the antiferromagnetic IrMn was obtained sufficient to bias the top layer of Py with a strong exchange bias field of at least $145 \mathrm{Oe}$, with no need for further annealing. The base pressure was $2.2 \times 10^{-7}$ Torr and the working pressures of Ar were 3.0 mTorr for deposition of the Py layer and 5.5 mTorr for deposition of the IrMn layer. ${ }^{20}$

The magnetic properties of the samples were measured by the Magneto-Optical Kerr Effect (MOKE), Ferromagnetic Resonance (FMR), and Brillouin Light Scattering (BLS) techniques. The MOKE measurements were carried out on a home-made set up. The He-Ne $(632.8 \mathrm{~nm})$ laser beam (10 $\mathrm{mW}$ ) was modulated by a photoelastic modulator working at $50 \mathrm{kHz}$. Magnetic hysteresis loops were obtained in the longitudinal configuration, with the light polarized along the dc magnetic field in the film plane. Figure 1(b) shows a MOKE measurement in the $\mathrm{Ni}_{81} \mathrm{Fe}_{19}(7.5 \mathrm{~nm}) / \mathrm{Ir}_{20} \mathrm{Mn}_{80}(35 \mathrm{~nm})$ sample exhibiting the characteristic shift of the hysteresis loop $\left(H_{E}\right)$. The FMR data were taken with a home-made set up operating at $9.4 \mathrm{GHz}$ at room temperature and a $1.2 \mathrm{kHz}$ magnetic field modulation frequency to allow lock-in detection of the absorption derivative. The sample, mounted on the tip of an external goniometer, was introduced through a hole in the back wall of the cavity. Thus, it can be rotated to allow measurements of the in-plane resonance field $H_{\text {res }}$ as a function of the azimuthal angle [see Fig. 1(c)], which were determined by fitting the data with a Lorentzian derivative line shape [see the inset of Fig. 1(c)]. The BLS measurements were carried with a Sandercock TFP-1 tandem Fabry-Perot interferometer system operating in a $(2 \times 3)$-pass configuration, with automatic control and data acquisition made with a TFPDAS4 interface board and software package. We have used a single-mode-stabilized diode-pumped solid state laser, Spectra Physics model Excelsior-532-300, that uses frequency-doubling techniques, operating at $532 \mathrm{~nm}$, with power $300 \mathrm{~mW}$. This power is attenuated by filters, and the power impinging on the sample is reduced to $33 \mathrm{~mW}$. The laser beam is focused on the film surface by a $\mathrm{f} / 1.7$ camera lens, with the plane of incidence normal to both the film plane and the field at an incident angle of $43^{\circ}$. The backscattered radiation was frequency analyzed by a Fabry-Perot interferometer in tandem mode. Due to the measurement geometry, the BLS spectra are due to spin waves propagating at $90^{\circ}$ with respect to film plane. The samples were mounted on a goniometer that allows rotation in the plane direction while maintaining the applied magnetic field in the film plane and with no change in the laser beam incident angle. Figure 1(d) shows a typical frequency spectrum of the spin waves for the same sample in Figs. 1(b) and 1(c) at a fixed applied field of 200 Oe. The BLS spectra taken at different angles were fit to Lorentzian line shapes to obtain in-plane angular dependence of the frequency shift.

\section{MODEL}

To interpret the FMR and BLS data, we calculate the spin-wave dispersion relation for the FM/AF bilayer. The calculation is based on the torque equation of motion for the FM magnetization, $\partial \boldsymbol{M}_{F} / \partial t=-\gamma \boldsymbol{M}_{F} \times \boldsymbol{H}_{\text {eff }}$, where $\gamma$ is the absolute value of the gyromagnetic ratio and $\boldsymbol{H}_{\text {eff }}$ is the effective field acting on $\boldsymbol{M}_{F}$. Following the approach of Refs. 21-24, the dispersion relation is

$$
\begin{aligned}
\left(\frac{\omega}{\gamma}\right)^{2}= & {\left[\left(\mathcal{E}_{\phi_{F M} \phi_{F M}}-\frac{\mathcal{E}_{\phi_{F M} \phi_{A F}}^{2}}{\mathcal{E}_{\phi_{A F} \phi_{A F}}}+f_{2}(q)\right)\right.} \\
& \left.\times\left(\mathcal{E}_{\theta_{F M} \theta_{F M}}-\frac{\mathcal{E}_{\theta_{F M} \theta_{A F}}^{2}}{\mathcal{E}_{\theta_{A F} \theta_{A F}}}+f_{1}(q)\right)\right] \frac{1}{\left(t_{F M} M_{F M}\right)^{2}}
\end{aligned}
$$

where $t_{F M}$ is the FM layer thickness and the $\mathcal{E}_{i j}$ terms denote the second derivative of the free energy per unit area with respect to the angles $\left(\phi_{F M}, \theta_{F M}\right)$ and $\left(\phi_{A F}, \theta_{A F}\right)$ of the FM and AF sublattice (in contact with the FM layer) magnetizations, respectively. The dependence on the spin wave number $q$ is given by the functions $f_{1}(q)$ and $f_{2}(q)$

$$
\begin{gathered}
f_{1}(q)=-2 \pi M_{S} q t_{F M}+D q^{2}, \\
f_{2}(q)=2 \pi M_{S} q t_{F M} \cos ^{2}\left(\phi_{H}-\phi_{F M}\right)+D q^{2},
\end{gathered}
$$

where $D$ is the stiffness constant of the FM film and $M_{S}$ is its saturation magnetization. When deriving Eq. (1), we consider the dc magnetic field applied in the plane of the sample so we assumed that the magnetization of both layers is in the plane, i.e., $\theta_{A F}=\theta_{F M}=\pi / 2$. Note that in FMR, where $q=0, f_{1}(0)=f_{2}(0)=0$ so that Eq. (1) coincides with the result of Refs. 12 and 13.

The equilibrium position of the magnetizations at which the terms in Eq. (1) are evaluated was obtained from the energy minimization of the magnetic free energy per unit area, ${ }^{25}$ which considers the relevant terms to describe magnetic behavior

$$
\begin{aligned}
\mathcal{E}= & -\left[\boldsymbol{H} \cdot \boldsymbol{M}_{\boldsymbol{F M}}+K_{u}\left(\frac{\boldsymbol{M}_{F M} \cdot \hat{\boldsymbol{u}}_{U}}{M_{F M}}\right)^{2}\right. \\
& \left.-\left(2 \pi M_{F M}^{2}-\frac{K_{s}}{t_{F M}}\right)\left(\frac{\boldsymbol{M}_{F M} \cdot \hat{\boldsymbol{n}}}{M_{F}}\right)^{2}\right] t_{F M}-J_{E} \frac{\boldsymbol{M}_{F M} \cdot \boldsymbol{M}_{A F}}{M_{F M} \cdot M_{A F}} \\
& -\sigma_{W} \frac{\boldsymbol{M}_{A F} \cdot \hat{\boldsymbol{u}}_{p i n}}{M_{A F}}-K_{r a}\left(\frac{\boldsymbol{M}_{F M} \cdot \hat{\boldsymbol{h}}}{M_{F M}}\right)^{2}
\end{aligned}
$$




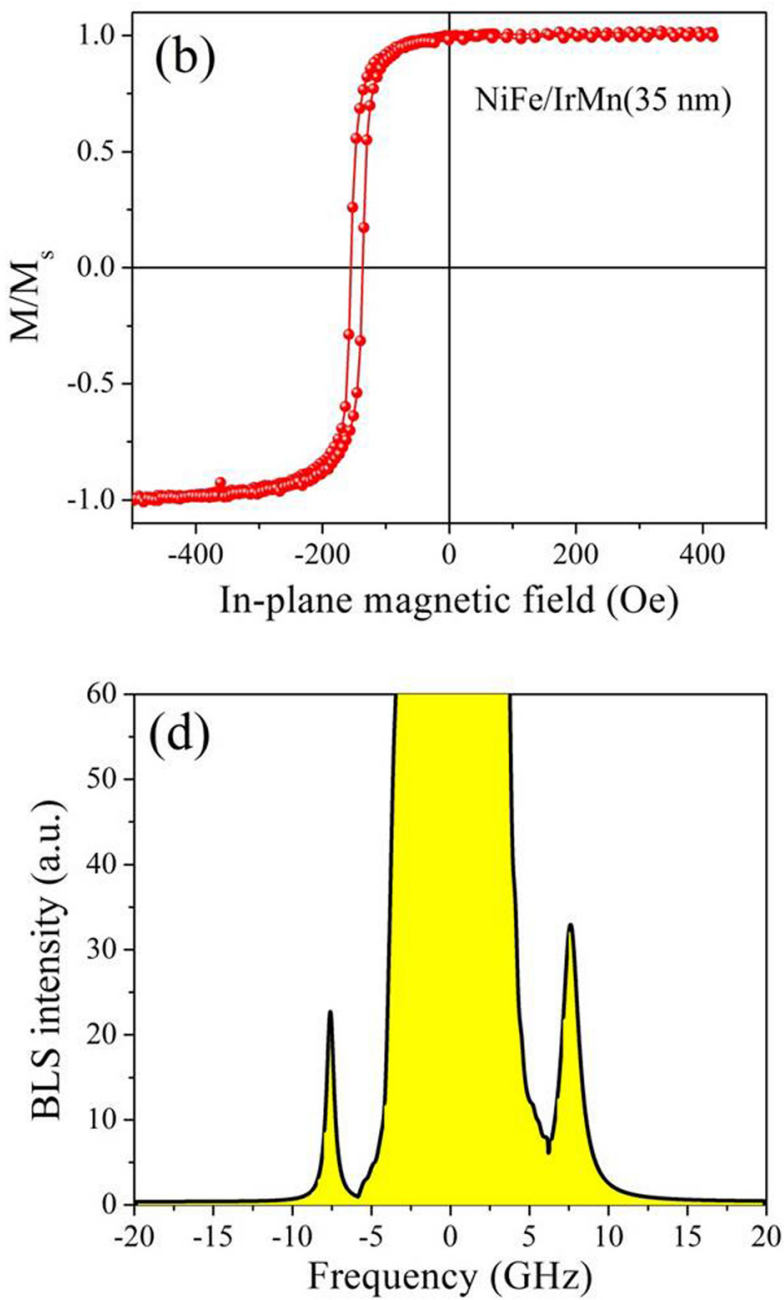

FIG. 1. (a) Coordinate system used to write the free magnetic energy and magnon dispersion relation. The uniaxial and unidirectional symmetry axes are parallel; (b) MOKE hysteresis loop of the $\mathrm{Ni}_{81} \mathrm{Fe}_{19} / \mathrm{Ir}_{20} \mathrm{Mn}_{80}\left(35 \mathrm{~nm}\right.$ ) bilayer measured with applied field parallel to $\hat{u}_{\text {pin }}$; (c) In-plane angular dependence of the resonance field. The solid line is the numerical fit that gives $H_{E}$ value. The inset is a field scan of FMR microwave absorption derivative spectra at $9.4 \mathrm{GHz}$. The solid line illustrates the fitting with a Lorentzian derivative by which resonance field is measured; (d) BLS photon counting. The larger central peak means the laser frequency and the two other peaks frequency shifted are the Stokes and anti-Stokes.

where the first three terms represent in order the Zeeman, uniaxial, and demagnetizing anisotropy energies with $K_{s}$ and $K_{u}$ being the surface and uniaxial anisotropy constants and $\hat{\boldsymbol{n}}$ the normal to the film plane. The fourth and fifth terms represent the interfacial coupling energy of the FM/AF bilayer, with exchange coupling constant $J_{E}>0$ and the AF domainwall energy created at the AF layer by the stable grains due to FM magnetization rotation. ${ }^{26}$ The last term in Eq. (3) is the rotatable anisotropy in the form introduced by Geshev, ${ }^{13}$ and takes into account the antiferromagnetic unstable grain contribution to the ferromagnetic magnetization rotation.

As pointed out, in this work the applied magnetic field direction lies on film plane, and consequently, as our samples present in-plane uniaxial anisotropy, the equilibrium position of magnetization can be calculated considering all vectors parallel to the film plane. Figure 1(a) shows the coordinate system used to represent the vectors in Eq. (3), where $\boldsymbol{H}$, $\boldsymbol{M}_{F M}$, and $\boldsymbol{M}_{A F}$ are the applied magnetic field, the magnetization of the FM layer, and the magnetization of the AF sublattice in atomic contact with FM, respectively. The unit vectors $\hat{\boldsymbol{u}}_{U}, \hat{\boldsymbol{u}}_{\text {pin }}$, and $\hat{\boldsymbol{h}}$ represent the FM uniaxial anisotropy direction, the AF pinning direction created by the stable grains, and the applied magnetic field direction, respectively.

From Eqs. (1) and (3), the magnetic excitation frequencies are given by

$$
\begin{aligned}
\left(\frac{\omega}{\gamma}\right)^{2}= & \left\{H \cos \left(\phi_{H}-\phi_{F M}^{0}\right)+H_{U} \cos 2 \phi_{F M}^{0}\right. \\
& \left.+H_{R A} \cos \left[2\left(\phi_{H}-\phi_{F M}^{0}\right)\right]+f_{2}(q)+\mathcal{H}_{2}\right\} \\
& \times\left\{H \cos \left(\phi_{H}-\phi_{F M}^{0}\right)+4 \pi M_{e f f}+H_{U} \cos ^{2} \phi_{F M}^{0}\right. \\
& \left.+H_{R A} \cos ^{2}\left(\phi_{H}-\phi_{F M}\right)+f_{1}(q)+\mathcal{H}_{1}\right\},
\end{aligned}
$$

where

$$
\mathcal{H}_{1}=\frac{H_{W} \cos \phi_{A F}^{0} \cos \left(\phi_{F M}^{0}-\phi_{A F}^{0}\right)-H_{E} \sin ^{2}\left(\phi_{F M}^{0}-\phi_{A F}^{0}\right)}{\frac{H_{W}}{H_{E}} \cos \phi_{A F}^{0}+\cos \left(\phi_{F M}^{0}-\phi_{A F}^{0}\right)}
$$


and

$$
\mathcal{H}_{2}=\frac{H_{W} \cos \phi_{A F}^{0} \cos \left(\phi_{F M}^{0}-\phi_{A F}^{0}\right)}{\frac{H_{W}}{H_{E}} \cos \phi_{A F}^{0}+\cos \left(\phi_{F M}^{0}-\phi_{A F}^{0}\right)} .
$$

Here, $H$ is the applied magnetic field strength, $H_{U}$ $=2 K_{u} / M_{F M}$ is the uniaxial FM anisotropy field, $H_{E}$ $=J_{E} / t_{F M} M_{F M}$ is the exchange bias interface coupling field, $H_{R A}=2 K_{r a} / t_{F M} M_{F M}$ is the rotatable anisotropy field, and $H_{W}=\sigma_{w} / t_{F M} M_{F M}$ is the domain wall field. $M_{e f f}$ is the effective magnetization defined by $4 \pi M_{e f f}=4 \pi M_{F M}-H_{S}$, where $H_{S}=2 K_{S} / t_{F M} M_{F M}$ is the surface anisotropy field. The angles $\phi_{F M}^{0}$ and $\phi_{A F}^{0}$ define the equilibrium position of the magnetizations of FM and AF layers, respectively. They are numerically obtained by a minimization process applied to Eq. (3) and are used directly to interpret the experimental data.

The BLS frequency shift is given by Eq. (4) with fixed $H$ and $q=2 k_{L} \sin \alpha=16 \mu \mathrm{m}^{-1}$, where $k_{L}$ is the laser light wavenumber and $\alpha=43^{\circ}$ the incident angle in the backscattering geometry. The FMR resonance field is obtained from Eq. (4) with $q=0$ (the uniform mode) at a fixed frequency of $\omega / 2 \pi=9.4 \mathrm{GHz}$.

\section{RESULTS AND DISCUSSION}

Figure 2 shows the rotatable $\left(H_{R A}\right)$ and exchange bias $\left(H_{E}\right)$ field strengths obtained from the fit of the FMR data for a larger series of samples as explained in Sec. II and showed elsewhere. ${ }^{12}$ In order to investigate the applied field dependence of $H_{R A}$ and $H_{E}$ by means of the BLS technique, we choose three representative samples: (i) $\mathrm{Ni}_{81} \mathrm{Fe}_{19} /$ $\mathrm{Ir}_{20} \mathrm{Mn}_{80}(5.0 \mathrm{~nm})$ with exchange bias field, $H_{E} \approx 0 \mathrm{Oe}$ and maximum rotatable field of $H_{R A}=408$ Oe for which almost all AF grains, behave as unstable, contribute only to the rotatable anisotropy field; (ii) $\mathrm{Ni}_{81} \mathrm{Fe}_{19} / \mathrm{Ir}_{20} \mathrm{Mn}_{80}(10.0 \mathrm{~nm}$ ) having stable $\mathrm{AF}$ grains that contribute to a small exchange

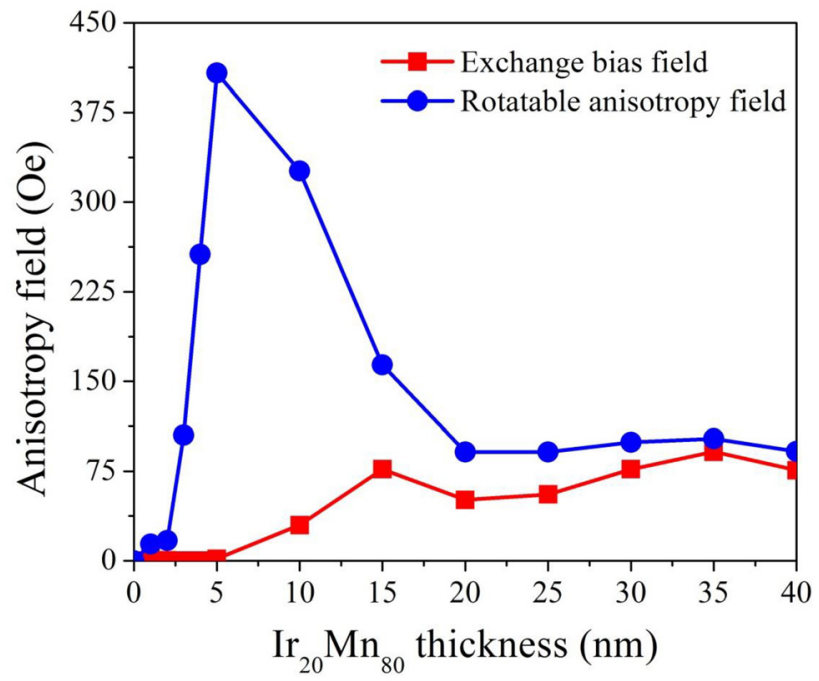

FIG. 2. Antiferromagnetic thickness dependency of exchange bias $H_{E}$ (red symbols) and rotatable field $H_{R A}$ (blue symbols). The values were extracted from the fit of the in-plane angle dependency of the ferromagnetic resonance field. Solid lines are guides for eyes.

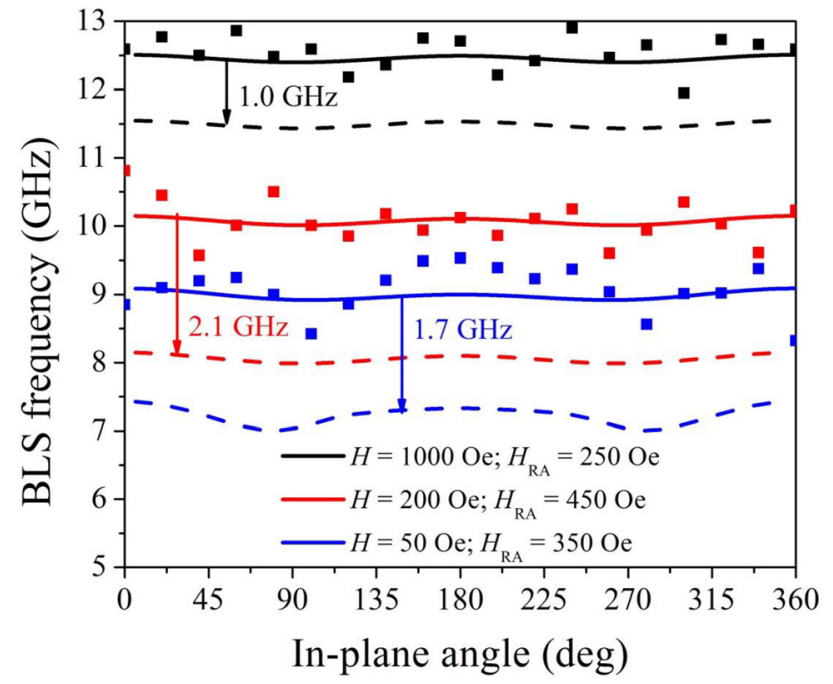

FIG. 3. Angular dependence of the BLS frequency measured with $H=50 \mathrm{Oe}$, (blue squares), $200 \mathrm{Oe}$ (red squares), and $1000 \mathrm{Oe}$ (black squares) for the sample $\mathrm{Ni}_{81} \mathrm{Fe}_{19} / \mathrm{Ir}_{20} \mathrm{Mn}_{80}(5.0 \mathrm{~nm})$. Solid lines represent the best fits. Solid lines are the result of the calculations using Eq. (4) with $H_{R A}$ and $H_{E}$ values as fitting parameters. Dashed lines represent the numerical calculation making $H_{R A}=0$. As the arrows indicate, frequency downshift for all applied field (positive RA) depends on the applied field.

bias field of $H_{E} \approx 30$ Oe but still with a greater number of unstable AF grains giving rise to a substantial rotatable field of $H_{R A}=326$ Oe and (iii) $\mathrm{Ni}_{81} \mathrm{Fe}_{19} / \mathrm{Ir}_{20} \mathrm{Mn}_{80}(35.0 \mathrm{~nm}$ ) that is the sample in the saturation regime of $H_{R A}$ and $H_{E}$ values under FMR conditions. It represents the region for $t_{A F}$ $\geq 20 \mathrm{~nm}$ in which stable/unstable AF grain distribution is almost constant, and as a consequence, the values of $H_{R A}$ and $H_{E}$ remain constant.

Figure 3 shows the in-plane angular dependence of the BLS frequency shift for the sample $\mathrm{Ni}_{81} \mathrm{Fe}_{19} / \mathrm{Ir}_{20} \mathrm{Mn}_{80}(5.0 \mathrm{~nm})$ measured at three different applied fields of $H=50,200$, and 1000 Oe. For the numerical calculations, the effective magnetization value $4 \pi M_{\text {eff }}=9.38 \mathrm{kG}$ was previously extracted from the fit of the FMR experimental data corresponding to the $\mathrm{Ni}_{81} \mathrm{Fe}_{19}(7.5 \mathrm{~nm})$ thin film, where $\gamma=17.6 \mathrm{GHz} / \mathrm{kOe}$, $4 \pi M_{S}=11.2 \mathrm{kG}$, and $D=2.0 \times 10^{7} \mathrm{Oe} \cdot \AA^{2}$. The dashed lines are the results of numerical calculation using Eq. (4) but making $H_{R A}=0$, that is, disregarding the rotatable anisotropy. As expected for polycrystalline $\mathrm{AF}$ in $\mathrm{FM} / \mathrm{AF}$ bilayers, $H_{R A} \neq 0$ and numerical data exhibit an overall downshift of a few $\mathrm{GHz}$ when compared to the experimental one. Table I summarizes the MOKE results. It shows that at applied fields of 200 Oe and 1000 Oe the Py film is saturated for all in-plane angles. Therefore, at first sight we should expect no change in the $H_{R A}$ value with $H$. However, as is clear from the size of

TABLE I. Values of exchange bias field $\left(H_{E}\right)$, saturation field $\left(H_{s a t}\right)$, and coercivity, obtained from MOKE measurements.

\begin{tabular}{|c|c|c|c|c|c|}
\hline \multirow[b]{2}{*}{$t_{A F}(\mathrm{~nm})$} & \multirow[b]{2}{*}{$H_{E}(\mathrm{Oe})$} & \multicolumn{2}{|c|}{ Easy axis } & \multicolumn{2}{|c|}{ Hard axis } \\
\hline & & $H_{\text {sat }}(\mathrm{Oe})$ & Coercivity (Oe) & $H_{\text {sat }}(\mathrm{Oe})$ & Coercivity (Oe) \\
\hline 5.0 & 0 & 90 & 154 & 180 & 47 \\
\hline 10.0 & 130 & 307 & 145 & 600 & 28 \\
\hline 35.0 & 140 & 285 & 11 & 500 & 16 \\
\hline
\end{tabular}



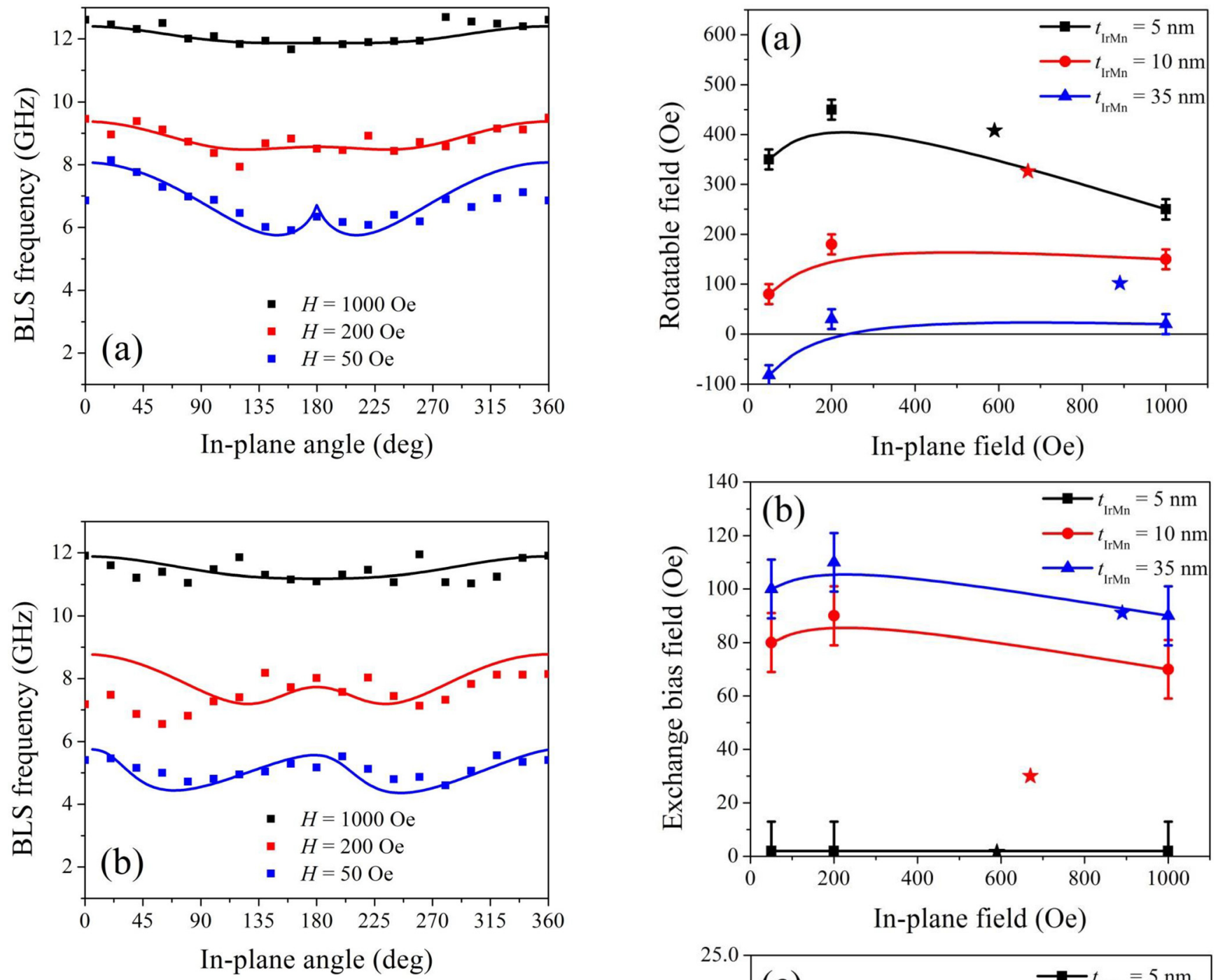

FIG. 4. Angular dependence of the BLS frequency measured with $H=50 \mathrm{Oe}$, (blue squares), $200 \mathrm{Oe}$ (red squares), and $1000 \mathrm{Oe}$ (black squares) for the samples; (a) $\mathrm{Ni}_{81} \mathrm{Fe}_{19} / \mathrm{Ir}_{20} \mathrm{Mn}_{80}\left(10.0 \mathrm{~nm}\right.$ ) and (b) $\mathrm{Ni}_{81} \mathrm{Fe}_{19} /$ $\mathrm{Ir}_{20} \mathrm{Mn}_{80}(35.0 \mathrm{~nm})$. Solid lines are fits obtained from Eq. (4).

the arrows in Fig. 3 the isotropic downshift of the BLS frequency depends on the applied field strength. Solid lines are the results of the calculation using Eq. (4) with $H_{R A}$ and $H_{E}$ values as fitting parameters.

Figures 4(a) and 4(b) show the numerical fits of the BLS data for the samples $\mathrm{Ni}_{81} \mathrm{Fe}_{19} / \mathrm{Ir}_{20} \mathrm{Mn}_{80}(10.0 \mathrm{~nm})$ and $\mathrm{Ni}_{81} \mathrm{Fe}_{19} / \mathrm{Ir}_{20} \mathrm{Mn}_{80}(35.0 \mathrm{~nm})$, respectively. The anisotropy fields extracted from the best fits to the experimental data are shown in Fig. 5. The field error bars were calculated considering a $5 \%$ increase on the square-chi of the magnon frequency versus in-plane angle numerical fit. There are no error bars for $H_{U}$ values because it was considered a fixed parameter in BLS data fitting, which were obtained from the $H_{\text {res }}$ versus in-plane angle fit. The solid lines are guide to the eyes and star points show the FMR results obtained from the fit of the in-plane dependence of the resonance field [as in the inset in Fig. 1(c)] using Eq. (4) with $q=0$ at a fixed frequency of $\omega / 2 \pi=9.4 \mathrm{GHz}$. Figures 5(a) and 5(b) show the rotatable anisotropy $H_{R A}$ and exchange $H_{E}$ fields as a function of the applied field $H$ during the BLS measurement. As

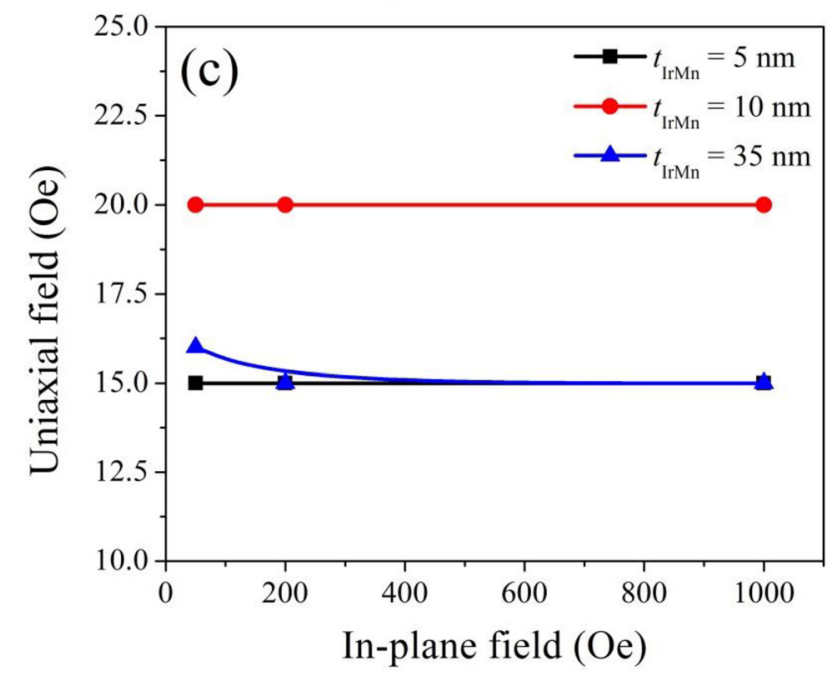

FIG. 5. Anisotropy fields obtained from the numerical fits of the BLS frequency vs. angle showed in Fig. 4 for the three field values of $H=50 \mathrm{Oe}$, $200 \mathrm{Oe}$, and $1000 \mathrm{Oe}$. (a) Rotatable anisotropy field $\left(H_{R A}\right)$ vs. $H$; (b) Exchange bias field $\left(H_{E}\right)$ vs. $H$; (c) Uniaxial anisotropy field $\left(H_{U}\right)$ vs. $H$. Star symbols are the results obtained from the FMR measurements at the corresponding resonant field. Solid lines are guides for the eyes.

we can observe, both fields clearly exhibit a non-monotonic dependence with $H$. Therefore, we should expect that the number and distribution of stable and unstable AF grains could be modified by the external field. 
To explain these experimental facts, we have considered the coexistence of parallel and antiparallel coupling at the FM/AF interface. This actually agrees with works on the same system. ${ }^{8,19,27}$ In this sense, following Schafer et al. ${ }^{19}$ we consider the presence of AF grains at the FM/AF interface that could be ferromagnetically (FMC) or antiferromagnetically (AFC) coupled to the FM magnetization. Each grain is classified as unstable (either superparamagnetic or rotatable), or stable (pinned), and both differ in number and anisotropy energy. Since, the anisotropy energies of each FMC or AFC grain differ, they rotate at different field strengths. Thereby, the lower the grain energy, the smaller the field required to rotate it.

The role played by the two types of grains, FMC or $\mathrm{AFC}$ at the interface, being these pinned or rotated is schematically explained through the block diagram of Fig. 6. At zero field, both grains are stable because there is no torque acting on their magnetizations. In our BLS experiment, the sample is rotated in a constant applied field $H \neq 0$ and the magnetization orientation of the FMC and AFC grains is ruled by the competition between their anisotropy energy and the interface exchange coupling. While pinned, both grains contribute only for $\mathrm{EB}$, which can be positive or negative as depicted in Fig. 6. When rotated, they lose the pinning, cease to be responsible for $H_{E}$ and start to contribute to $H_{R A}$, which also can be positive or negative, according to Fig. 6. Then, in order to explain Figs. 5(a) and 5(b) we follow this approach.
Next we analyze the data for the several samples: the sample $\mathrm{Ni}_{81} \mathrm{Fe}_{19} / \mathrm{Ir}_{20} \mathrm{Mn}_{80}(35.0 \mathrm{~nm})$ presents negative $H_{R A}$ at low applied field strength [blue symbols in Fig. 5(a)]. To explain that, consider that FMC grains exceed AFC ones in number and in anisotropy energy. This is supported by the negative value of $H_{E}$ obtained in the MOKE measurements and also by the concave bell shape of the FMR resonance field curve [see Fig. 1(c)]. Then, for $H=50$ Oe those AFC grains having lowest energy rotate contributing to a negative value of $H_{R A}$. That is, as they are antiferromagnetically coupled, once they rotate their magnetizations are no longer oriented along the energy minimum, the curvature of $\mathcal{E}$ in Eq. (3) is decreased and the magnon frequency decreases. The FMC grains having a higher pinning energy do not change their state at this field and only contribute for $H_{E}$. When the field further increases, an increasing number of FMC grains begin to rotate contributing to a positive value of $H_{R A}$ as depicted in Fig. 6. In this case, its magnetization is oriented along a minimum, the curvature of $\mathcal{E}$ is increased and the magnon frequency increases resulting in the frequency up-shift observed. This is what is observed between 50 Oe and 200 Oe for this sample. For fields above this value, the process saturates and the rotatable field strength remains roughly constant.

The hypothesis also explains the $H_{E}$ dependence with applied field shown in Fig. 5(b) for the same sample $\mathrm{Ni}_{81} \mathrm{Fe}_{19} / \mathrm{Ir}_{20} \mathrm{Mn}_{80}(35.0 \mathrm{~nm}$ ) (blue symbols). Note that FMC grains contribute to increases $H_{E}$ in FMR and BLS, whereas AFC ones decrease it. Between 50 Oe and $200 \mathrm{Oe}$, the



FIG. 6. Scheme of the magnetization orientation of the stable and unstable AF grains. Both ferromagnetically (FMC) and antiferromagnetically coupled (AFMC) grains to the FM magnetization are considered. The third row depicts common experimental manifestation of the exchange bias and rotatable anisotropy. The last row shows a schematic representation of magnetic energy considering only the exchange, uniaxial, and Zeeman contributions. 
rotated AFC grains cease to contribute to $H_{E}$. They become unstable and consequently the proportion of FMC/AFC grains favors to the increase of $H_{E}$. When all AFC grains have rotated, at high magnetic fields $(200 \mathrm{Oe}-1000 \mathrm{Oe}), H_{E}$ should remain constant, as observed.

For the sample $\mathrm{Ni}_{81} \mathrm{Fe}_{19} / \mathrm{Ir}_{20} \mathrm{Mn}_{80}(10.0 \mathrm{~nm}$ ) (red symbols) the situation is analogous. The difference is that as we can infer from Fig. 2, for this AF thickness there must be more unstable FMC grains and at low applied fields these contribute to positive $H_{R A}$ as observed in Fig. 5(a). When the external field strength is raised, the amount of unstable grains increases contributing to the slight increase of $H_{R A}$. For Fig. 5(b), we have the same explanation as before. Initially, stable $\mathrm{AFC}$ grains cease to contribute to the $H_{E}$ value. As field increases $(H>200 \mathrm{Oe})$, the process saturates leaving $H_{E}$ roughly constant.

For the sample $\mathrm{Ni}_{81} \mathrm{Fe}_{19} / \mathrm{Ir}_{20} \mathrm{Mn}_{80}(5.0 \mathrm{~nm})$, at the $\mathrm{EB}$ threshold (see Fig. 2), the behavior of $H_{R A}$ is different [Fig. 5(a), black symbols]. In this case, all grains, either FMC or AFC grains, must be unstable and consequently are not capable to sustain $H_{E}$ [see Fig. 5(b), black symbols]. This could explain the $H_{R A}$ dependence with the applied field $H$. Considering only the Zeeman, uniaxial, and exchange energies there are two energy minima, parallel $\left(\mathcal{E}_{\|}\right)$or antiparallel $\left(\mathcal{E}_{\nVdash}\right)$ to $H$ with an energy barrier between them (see scheme in Fig. 6). At low field values, the energy barrier is almost the same in either direction and $H_{R A}$ must be approximately zero. When $H$ is increased, grains FM coupled in the state $\mathcal{E}_{\nVdash}^{(F M C)}$ have an energy barrier lower than those AF coupled, jump to the $\mathcal{E}_{\|}^{(F M C)}$ minimum contributing to a positive $H_{R A}$. This explains the increase of $H_{R A}$ between 50 Oe and 200 Oe. After that, when the external field further increases, those grains $\mathrm{AF}$ coupled initially in the state $\mathcal{E}_{\nVdash}^{(A F M C)}$ will be dragged to the $\mathcal{E}_{\|}^{(A F M C)}$ contributing now to a negative $H_{R A}$. This finally explains the considerable decrease in $H_{R A}$ at high fields. Figure 5(c) shows the uniaxial anisotropy field obtained from the fits of BLS and FMR data. Since it is a magnetocrystalline anisotropy contribution, we do not expect any applied field dependence, and therefore, changes on the FM during the growth are not responsible for the reported results.

Finally, it is worth to comment on the disagreement between the results for EB obtained with different techniques, MOKE, FMR (stars in Fig. 5), and BLS (squares in Fig. 5), pointed out some time ago in Ref. 28 and observed in Ref. 29. As we can observe, in our samples $H_{E}^{(M O K E)}>H_{E}^{(F M R)}$, in agreement with the condition that the FMR field is high enough to induce antiparallel-parallel reorientation. On the other hand, although FMR and BLS are both perturbative techniques, in the former external perturbation plays an important role because the sample is located in a position of maximum rf field inside the resonant cavity. In this sense, we would expect, as obtained, that $H_{E}^{(F M R)}<H_{E}^{(B L S)}\left(H_{R A}^{(F M R)}>H_{R A}^{(B L S)}\right)$ due to the antiparallel-parallel reorientation.

\section{CONCLUSIONS}

We used the backscattering Brillouin light scattering set up to measure magnon frequencies as a function of the in- plane angle for FM/AF coupled for various magnetic field intensities. From the comparison between numerical fit and experimental data, we found that the stable and unstable antiferromagnetic grains distribution has a strong field dependency. We reported a novel behavior of the rotatable anisotropy in EB bilayers of $\mathrm{Ni}_{81} \mathrm{Fe}_{19}(7.5 \mathrm{~nm}) / \mathrm{Ir}_{20} \mathrm{Mn}_{80}\left(t_{A F}\right)$ showing that the rotatable and exchange anisotropies have a non-monotonic dependence with the applied field. The experimental results were explained considering the coexistence of parallel and antiparallel coupling at the FM/AF interface. These results along with a new approach to study the EB phenomenon might be useful when designing magnetic sensors, based on FM/AF bilayers, to work in different magnetic field environments.

\section{ACKNOWLEDGMENTS}

This research was supported in Brazil by Conselho Nacional de Desenvolvimento Científico e Tecnológico (CNPq), Coordenação de Aperfeiçoamento de Pessoal de Nível Superior (CAPES), Financiadora de Estudos e Projetos (FINEP), and Fundação de Amparo à Ciência e Tecnologia do Estado de Pernambuco (FACEPE), in Chile by Fondo Nacional de Desarrollo Científico y Tecnológico (FONDECYT) No. 1170723, and in Mexico by Consejo Nacional de Ciencia y Tecnología (CONACYT). Authors M. Arana and F. Estrada thank CNPq for the grants of their Post-Doctoral fellowship.

${ }^{1}$ W. H. Meiklejohn and C. P. Bean, Phys. Rev. 102, 1413 (1956).

${ }^{2}$ B. Dieny, V. S. Speriosu, S. S. P. Parkin, B. A. Gurney, D. R. Wilhoit, and D. Mauri, Phys. Rev. B 43, 1297 (1991).

${ }^{3}$ J. Nogués, J. Sort, V. Langlais, V. Skumryev, S. Suriñach, J. S. Muñoz, and M. D. Baró, Phys. Rep. 422, 65 (2005).

${ }^{4}$ T. Kosub, M. Kopte, R. Hühne, P. Appel, B. Shields, P. Maletinsky, R. Hübner, M. O. Liedke, J. Fassbender, O. G. Schmidt, and D. Makarov, Nat. Commun. 8, 13985 (2017).

${ }^{5}$ O. Gomonay, T. Jungwirth, and J. Sinova, Phys. Status Solidi Rapid Res. Lett. 11(4), 1700022 (2017).

${ }^{6}$ J. Sinova and I. Žutić, Nat. Mater. 11, 368 (2012).

${ }^{7}$ J. Nogués, D. Lederman, T. J. Moran, and I. K. Schuller, Phys. Rev. Lett. 76, 4624 (1996).

${ }^{8}$ S. K. Mishra, F. Radu, H. A. Dür, and W. Eberhardt, Phys. Rev. Lett. 102, 177208 (2009).

${ }^{9}$ H. Fulara, S. Chaudhary, S. C. Kashyap, and D. K. Pandya, J. Appl. Phys. 110, 093916 (2011).

${ }^{10}$ R. D. McMichael, M. D. Stiles, P. J. Chen, and W. F. Egelhoff, Jr., Phys. Rev. B 58, 8605 (1998).

${ }^{11}$ M. D. Stiles and R. D. McMichael, Phys. Rev. B 59, 3722 (1999).

${ }^{12}$ R. L. Rodríguez-Suárez, L. H. Vilela-Leão, T. Bueno, A. B. Oliveira, J. R. L. de Almeida, P. Landeros, S. M. Rezende, and A. Azevedo, Phys. Rev. B 83, 224418 (2011).

${ }^{13}$ J. Geshev, L. G. Pereira, and J. E. Schmidt, Phys. Rev. B 66, 134432 (2002).

${ }^{14}$ M. D. Stiles and R. D. McMichael, Phys. Rev. B 60, 12950 (1999).

${ }^{15}$ M. D. Stiles and R. D. McMichael, Phys. Rev. B 63, 064405 (2001).

${ }^{16}$ S. Nicolodi, L. G. Pereira, A. Harres, G. M. Azevedo, J. E. Schmidt, I. García-Aguilar, N. M. Souza-Neto, C. Deranlot, F. Petroff, and J. Geshev, Phys. Rev. B 85, 224438 (2012).

${ }^{17}$ P. G. Barreto, M. A. Sousa, F. Pelegrini, W. Alayo, F. J. Litterst, and E. Baggio-Saitovitch, Appl. Phys. Lett. 104, 202403 (2014).

${ }^{18}$ M. A. Sousa, F. Pelegrini, W. Alayo, J. Quispe-Marcatoma, and E. Baggio-Saitovitch, Phys. B: Condens. Matter 450, 167 (2014).

${ }^{19}$ D. Schafer, P. L. Grande, L. G. Pereira, G. M. Azevedo, A. Harres, M. A. de Sousa, P. Pelegrini, and J. Geshev, J. Appl. Phys. 117, 215301 (2015).

${ }^{20}$ A. J. Devasahayam, P. J. Sides, and M. H. Kryder, J. Appl. Phys. 83, 7216 (1998).

${ }^{21}$ B. Hillebrands, Phys. Rev. B 41, 530 (1990). 
${ }^{22}$ J. F. Cochran, J. Rudd, W. B. Muir, B. Heinrich, and Z. Celinski, Phys. Rev. B 42, 508 (1990).

${ }^{23}$ P. Kabos, C. E. Patton, M. O. Dima, and D. B. Church, J. Appl. Phys. 75, 3553 (1994).

${ }^{24}$ S. M. Rezende, C. Chesman, M. A. Lucena, A. Azevedo, F. M. de Aguiar, and S. S. P. Parkin, J. Appl. Phys. 84, 958 (1998).

${ }^{25}$ A. B. Oliveira, R. L. Rodriguez-Suarez, S. Michea, H. Vega, A. Azevedo, S. M. Rezende, C. Aliaga, and J. Denardin, J. Appl. Phys. 116, 033910 (2014).
${ }^{26}$ D. Mauri, H. C. Siegmann, P. S. Bagus, and E. Kay, J. Appl. Phys. 62, 3047 (1987).

${ }^{27}$ G. Salazar-Alvarez, J. J. Kavich, J. Sort, A. Muarza, S. Stepanow, A. Potenza, H. Marchetto, S. S. Dhesi, V. Naltz, B. Dieny, A. Weber, L. J. Heyderman, J. Nogués, and P. Gambardella, Appl. Phys. Lett. 95, 012510 (2009).

${ }^{28}$ H. Xi, R. M. White, and S. M. Rezende, Phys. Rev. B 60, 14837 (1999).

${ }^{29}$ J. R. Fermin, M. A. Lucena, A. Azevedo, F. M. de Aguiar, and S. M. Rezende, J. Appl. Phys. 87, 6421 (2000). 